\title{
$M$-Connected Coverage Problem in Wireless Sensor Networks
}

\author{
S. Mini, ${ }^{1}$ Siba K. Udgata, ${ }^{1}$ and Samrat L. Sabat ${ }^{2}$ \\ ${ }^{1}$ Department of Computer and Information Sciences, University of Hyderabad, Hyderabad 500046, India \\ ${ }^{2}$ School of Physics, University of Hyderabad, Hyderabad 500046, India
}

Correspondence should be addressed to Siba K. Udgata, udgatacs@uohyd.ernet.in

Received 22 December 2011; Accepted 9 January 2012

Academic Editors: J.-F. Myoupo and Y.-C. Wang

Copyright (C 2012 S. Mini et al. This is an open access article distributed under the Creative Commons Attribution License, which permits unrestricted use, distribution, and reproduction in any medium, provided the original work is properly cited.

\begin{abstract}
Solving coverage problem alone is not adequate in a wireless sensor network, since data has to be transmitted to the base station. This leads to the lookout for an energy efficient method to solve connected coverage problem. This paper addresses $M$-connected (each sensor node will have at least $M$ other sensor nodes within its communication range) target coverage problem in wireless sensor networks, where the required level of connectivity and coverage may be high or low as required. We propose a heuristic for $M$-connected target coverage problem, where initially a cover is decided and later on it is checked for $M$-connectivity. $M$-connectivity for simple coverage, $k$-coverage, and $Q$-coverage is focussed on in this paper. We use a Low-Energy Adaptive Clustering Hierarchy (LEACH) inspired model, where a cluster is considered as a set of sensor nodes satisfying $M$-connectivity and required level of coverage. It is enough if one among these nodes transmits the monitored information to the base station. When the required level of coverage are high, chances of nodes being connected in the cover is high. Simulation results show that our proposed method can achieve better results than Communication Weighted Greedy Cover (CWGC).
\end{abstract}

\section{Introduction}

In recent years, there has been considerable progress in wireless sensor networks (WSNs) research. A WSN can be structured or unstructured [1]. An unstructured WSN is one that contains a dense collection of sensor nodes and sensor nodes may be deployed randomly into the field. Hence, network maintenance such as managing connectivity and detecting failures is difficult. In a structured WSN, all or some of the sensor nodes are deployed in a preplanned manner. Among the major challenges in WSN are coverage, and connectivity problems. Coverage in a WSN needs to guarantee that the monitored region is completely covered with a high degree of reliability [1]. Connectivity scales the adequacy with which the nodes are able to communicate [2]. Coverage problem can be classified based on the region/object to be monitored [3] as area coverage problem and target coverage problem. Area coverage problem aims at monitoring/gathering information about an entire region. The target coverage problem concerns about monitoring a set of specific locations in the region.

Target coverage problems evolved in 3 stages; simple coverage, $k$-coverage and $Q$-coverage. With simple coverage, each target should be monitored by at least one sensor node. For compensating node failures or in case of monitoring with greater accuracy, simple coverage was not sufficient. This paved the way for $k$-coverage, where each target has to be monitored by at least $k$ sensor nodes, where $k$ is a predefined integer constant. $k$ coverage problem seems unfit for applications where targets need not essentially be monitored by the same number of sensor nodes. This leads to $Q$-coverage, where $T=\left\{T_{1}, T_{2}, \ldots, T_{n}\right\}$ should be monitored by $Q=\left\{q_{1}, q_{2}, \ldots, q_{n}\right\}$ number of sensor nodes such that target $T_{j}$ is monitored by at least $q_{j}$ number of sensor nodes, where $n$ is the number of targets and $1 \leq j \leq n$.

The coverage requirement depends on the application. Some applications require complete coverage at all times, whereas the coverage requirement can slightly be compromised for some other applications [4]. The time during which all the sensors can sense or communicate is limited because of irreplaceable batteries.

It is imperative to look into the connectivity aspect of WSNs, together with issues of coverage [5]. Two sensor nodes are said to be connected if they are located within the communication range of each another. Connectivity depends on the random distribution of nodes [6]. M-connectivity 


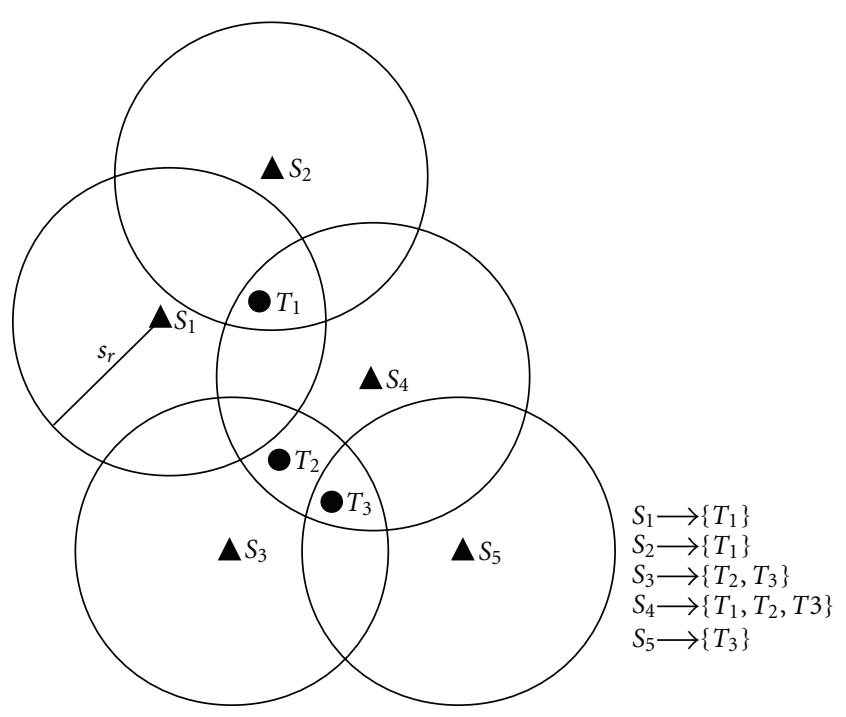

FIgURE 1: Nonconnected coverage.

implies that each node in the sensor cover (a subset of sensor nodes that meets required level of coverage) is connected to at least $M$ other sensor nodes in the same cover. Sensor deployment can either be deterministic or random. In deterministic deployment, coverage can be maximized as a result of optimal placement of sensor nodes. Random deployments are preferred when the region information is not known a priori. When sensor nodes are randomly deployed, few objects in the region may be densely covered and few may be sparsely covered. Figure 1 shows a sample random deployment where there are 3 targets $\left\{T_{1}, T_{2}, T_{3}\right\}$ and 5 sensor nodes $\left\{S_{1}, S_{2}, S_{3}, S_{4}, S_{5}\right\}$ to monitor the targets. All the targets are completely covered in the given deployment and it does not consider connectivity. Figure 2 shows a deployment where there are three sensor nodes $\left\{S_{1}, S_{2}, S_{3}\right\}$ to monitor two targets $\left\{T_{1}, T_{2}\right\}$. Here each sensor node has a sensing range $s_{r}$ and a communication range $c_{r}$. For nonconnected simple coverage to be satisfied, it is sufficient if $S_{1}$ and $S_{3}$ are activated. But for connected coverage, in addition to $S_{1}$ and $S_{3}, S_{2}$ should also be activated since $S_{2}$ connects $S_{1}$ and $S_{3}$.

Network lifetime, which is a vital performance parameter in WSNs that can be defined as the interval between the time when the network starts functioning and when the network dies. A WSN dies when it is not able to meet coverage and/or connectivity requirement and/or if it is deprived of sufficient battery power. Generally random deployment includes a large population of sensor nodes, and scheduling is a frequently used method to conserve energy [7]. Only minimum number of sensor nodes is activated to satisfy the coverage requirement and the remaining nodes are set to sleep for conserving energy. Hence these scheduling schemes prolong the lifetime of the sensor network. However, if more nodes are left to sleep, the WSN may be disconnected. This will affect data communication and transmission. Compared to nonconnected coverage, some extra nodes might have to be turned on to keep the network connected for satisfying connected coverage. Thus the network lifetime can

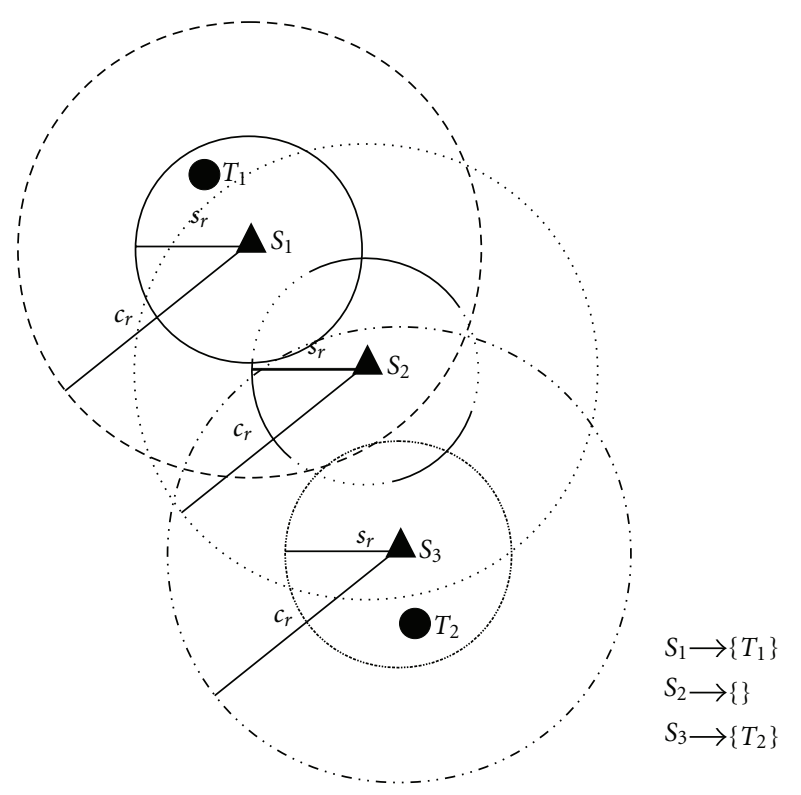

Figure 2: Connected coverage.

be augmented by scheduling sensor nodes such that only a subset of sensor nodes that meets the coverage and connectivity requirement needs to be active at a time.

Neither direct communication nor MTE (minimumtransmission-energy) routing is energy efficient [4]. Based on clustering, Heinzelman et al. [4] proposed LEACH which appears to be energy efficient. We define a cluster as a subset of sensor nodes which satisfies $M$-connectivity and required coverage level. We assume random deployment of sensor nodes, which is mostly used for inaccessible regions or in battlefield surveillance. We use binary sensing model, where if a target lies within the sensing region, it is always assumed to be detected with probability 1 otherwise with probability 0 . In this paper, we assume that all sensor nodes have the same initial battery power, sensing range, and communication range.

1.1. Importance. Sensor coverage is important while evaluating the effectiveness of a wireless sensor network. A lower coverage level (simple coverage) is enough for environmental or habitat monitoring [1] or applications like home security [8]. Higher degree of coverage ( $k$-coverage) will be required for some applications like target tracking to track the targets accurately [9], or if sensors work in a hostile environment such as battle fields or chemically polluted areas [8]. More reliable results are produced for higher degree of coverage which requires multiple sensor nodes to monitor the region/targets.

In some cases, for the same application, the coverage requirement may vary. For example, for forest fire detections, the coverage level may be low in rainy seasons, but high in dry seasons [8]. An example of $Q$-coverage is a video surveillance system deployed for monitoring hostile territorial area where some sensitive targets like a nuclear plant may need more sensors cooperate to ensure source redundancy for precise 
data [10]. In such applications, the nodes that are turned on should be connected to ensure proper data transmission. In case of simple connected coverage, where it is enough to have the nodes 1-connected, the network will be disconnected even if a single node fails. Hence it is important to have sufficient connectivity along with sufficient coverage. This paved the way for studying $M$-connected coverage problem where at least $M$-connectedness should exist within the nodes that are turned on, to guarantee the correctness of the information collected and also to ensure the reachability of the information at other nodes including the base station.

Rest of the paper is organized as follows: in Section 2, we briefly review some of the related work. The problem is formulated formally in Section 3. Section 4 presents the proposed method to solve the problem. Section 5 presents the simulation results. Concluding remarks in Section 6 end the paper.

\section{Related Work}

Several researchers have addressed target coverage problem without considering connectivity. Simple coverage problem has been addressed in [11-13]. $k$-coverage problem has been addressed in $[8,9,14]$. Q-coverage problem has been addressed in $[10,15-17]$. None of these address the connected coverage problem.

Zhou et al. [18] present a centralized approximation algorithm and a distributed version of the algorithm to solve connected $k$-coverage problem. The distributed priority algorithm is more efficient in applications where the query is executed for less than a few hundred times. For longer running queries, the distributed greedy algorithm is more efficient. Lu et al. [19] generalize the sleep/active mode by adjusting sensing range to maximize total number of rounds and present a distributed heuristic. A more generic connectivity condition that can be used even when the transmission range is less than twice the sensing range is considered. It deals with the case of scheduling sensors activity by selfconfiguring sensing ranges, in the environment where both discrete target coverage and network connectivity are satisfied. Gupta et al. [20] design and analyze algorithms for self-organization of a sensor network into an optimal logical topology in response to a query. A distributed version of the approximation algorithm that is run by the sensors in the network and results in a self-organization of the network into a topology involving a near-optimal number of sensors is also designed.

Zhao and Gurusamy [21] consider the Connected Target Coverage (CTC) problem with the objective of maximizing the network lifetime by scheduling sensors into multiple sets, each of which can maintain both target coverage and connectivity among all the active sensors and the sink. A faster heuristic algorithm based on the approximation algorithm called Communication Weighted Greedy Cover (CWGC) algorithm is designed and a distributed implementation of the heuristic algorithm is presented.

Some works propose deployment patterns such that the region of interest could be monitored with the required level of coverage and the network being connected. Some of these also aim at minimizing the number of sensor nodes that need to be placed. This is applicable only in the case of deterministic deployment. Bai et al. [22] investigate the problem of finding an optimal deployment pattern that achieves four connectivities and full coverage. A Diamond pattern, which can be viewed as a series of evolving patterns, is proposed.

Wang et al. [23] consider the sensing field as an arbitraryshaped region possibly with obstacles. The sensing field is partitioned into smaller subregions based on the shape of the field, and then sensors are deployed in these subregions such that the area is covered and connectivity is ensured. The number of sensors deployed is minimized using this approach.

Ammari and Das [24] compute the minimum sensor spatial density necessary for complete $k$-coverage of a sensor field. A tighter bound on network connectivity of $k$-covered WSNs, where the radius of the communication disks of sensors only needs to be at least equal to the radius of their sensing disks, is also derived.

Han et al. [25] investigate problems related to connected coverage in directional sensor networks where sensors only sense directionally and have a sector-like sensing range. Deployment patterns are proposed such that the location points/area is covered and forms a connected network. This also uses minimum number of directional sensors to form a connected network to cover a set of point locations and the entire target sensing area, respectively.

Gao and Zou [26] show that deployment of mobile nodes (Mules) improves the coverage and connectivity. Krause et al. [27] present an algorithm for sensor deployment which incurs minimal communication cost.

Kar and Banerjee [28] also address the problem of optimal node placement for ensuring connected coverage in sensor networks and propose two scenarios. The first scenario requires a complete area to be provided with connected coverage and the second scenario needs a given set of points in the region to be covered and connected.

In our model, sensors nodes are not deterministically deployed. Hence there is no provision of using an optimal deployment pattern to maintain coverage and connectivity. Optimal deployment patterns are used for deterministic deployment of sensor nodes to address area coverage or $k$ coverage of targets along with connectivity. Here since sensor nodes are dense and randomly deployed, the best way to prolong network lifetime is by scheduling the nodes such that the required level of coverage and connectivity is achieved with minimum number of sensor nodes.

Heinzelman et al. [4] propose LEACH which is based on clustering and also point out that using a direct communication protocol or MTE routing will not be optimal. Direct communication will require a large amount of transmission power from each node if the base station is far away from the nodes. In case of MTE routing, the nodes closest to the base station will be used to route a large number of data messages to the base station. Thus these nodes will die out quickly, causing the energy required to get the remaining data to the base station to increase and more nodes to die. With the use of clusters, LEACH was able to achieve large reduction 
in energy dissipation. Local computation in each cluster reduced the amount of data that must be transmitted to the base station.

Most of the works related to target coverage focus on direct communication or MTE routing, where the nodes closest to the base station are largely used. Unlike all of these, we focus on identifying a minimum-sized cluster which is $M$-connected and meets simple/k/Q-coverage requirement. Once this cluster is decided, routing information to the base station is done as in LEACH.

\section{Problem Definition}

Let us assume $m$ sensor nodes $\left\{S_{1}, S_{2}, \ldots, S_{m}\right\}$ randomly deployed to cover the area $R$ with $n$ targets $\left\{T_{1}, T_{2}, \ldots, T_{n}\right\}$. Each sensor node has an initial energy $E_{0}$, sensing radius $s_{r}$ and communication radius $c_{r}$. A sensor node $S_{i}, 1 \leq i \leq m$, is said to cover a target $T_{j}, 1 \leq j \leq n$, if the distance $d\left(S_{i}, T_{j}\right)$ between $S_{i}$ and $T_{j}$ is less than $s_{r}$. Two sensor nodes are said to be connected if one sensor node lies within the communication range of the other. The coverage matrix is defined as

$$
M_{i j}= \begin{cases}1 & \text { if } S_{i} \text { monitors } T_{j} \\ 0 & \text { otherwise }\end{cases}
$$

where $i=1,2, \ldots, m$ and $j=1,2, \ldots, n$.

The connectivity matrix is defined as

$$
C M_{i z}= \begin{cases}1 & \text { if } S_{i} \text { and } S_{z} \text { are connected and } i \neq z \\ 0 & \text { otherwise }\end{cases}
$$

where $i=1,2, \ldots, m$ and $z=1,2, \ldots, m$.

\subsection{M-Connected 1-Coverage Scheduling}

Definition 1. Given $m$ sensor nodes $S=\left\{S_{1}, S_{2}, \ldots, S_{m}\right\}$ with battery power $B=\left\{b_{1}, b_{2}, \ldots, b_{m}\right\}$, energy consumption rate $e_{i}$ for $S_{i}$ and $n$ targets $T=\left\{T_{1}, T_{2}, \ldots, T_{n}\right\}$, find a schedule $\left\{C_{1}, \ldots, C_{y}\right\}$ for time tick $\left\{t_{1}, \ldots, t_{y}\right\}$ such that for all ticks

(1) each target is covered by at least one of the sensor nodes,

(2) each sensor node in $C_{p}$ is connected to at least $M$ other nodes in $C_{p}$, where $1 \leq M \leq m$,

(3) network lifetime $\sum_{p=1}^{y} t_{p}$ is maximized.

\subsection{M-Connected $k$-Coverage Scheduling}

Definition 2. Given a set of sensor nodes $S=\left\{S_{1}, S_{2}, \ldots, S_{m}\right\}$ with battery power $B=\left\{b_{1}, b_{2}, \ldots, b_{m}\right\}$, energy consumption rate $e_{i}$ for $S_{i}$ and a target set $T=\left\{T_{1}, T_{2}, \ldots, T_{n}\right\}$, generate a schedule $\left\{C_{1}, \ldots, C_{y}\right\}$, for $\left\{t_{1}, \ldots, t_{y}\right\}$, such that for all ticks

(1) each target is covered by at least $k$ sensor nodes, $1 \leq$ $k \leq m$,

(2) each sensor node in $C_{p}$ is connected to at least $M$ other nodes in $C_{p}$, where $1 \leq M \leq m$,

(3) network lifetime $\sum_{p=1}^{y} t_{p}$ is maximized.

\subsection{M-Connected Q-Coverage Scheduling}

Definition 3. Given a set of sensor nodes $S=\left\{S_{1}, S_{2}, \ldots, S_{m}\right\}$ with battery power $B=\left\{b_{1}, b_{2}, \ldots, b_{m}\right\}$, energy consumption rate $e_{i}$ for $S_{i}$ and a target set $T=\left\{T_{1}, T_{2}, \ldots, T_{n}\right\}$, generate a schedule $\left\{C_{1}, \ldots, C_{y}\right\}$, for $\left\{t_{1}, \ldots, t_{y}\right\}$, such that for all ticks

(1) $T=\left\{T_{1}, T_{2}, \ldots, T_{n}\right\}$ is covered by at least $Q=$ $\left\{q_{1}, q_{2}, \ldots, q_{n}\right\}$ sensor nodes, where each target $T_{j}$, $1 \leq j \leq n$, is covered by at least $q_{j}$ sensor nodes,

(2) each sensor node in $C_{p}$ is connected to at least $M$ other nodes in $C_{p}$, where $1 \leq M \leq m$,

(3) network lifetime $\sum_{p=1}^{y} t_{p}$ is maximized,

1 -Coverage and $k$-coverage are special cases of $Q$-coverage where all $q_{j}=1$ and $q_{j}=k$, respectively.

\section{Proposed Method}

4.1. Cover Formation. Initially, a cover is computed without looking into connectivity. There may be different ways to generate sensor covers if the network has nodes which make all the targets $1 / k / Q$ covered. We use a priority-based method to compute the covers. The priority of sensor nodes is calculated based on the remaining battery power. The more the remaining battery power of a sensor node, the higher the priority of the sensor node. In the order of priority, if any new sensor node contributes to $1 / k / Q$ coverage requirement, it will be added to the cover set. In general, a sensor node $S_{i}$ can be added to a cover set Cov_S if and only if

(1) for simple coverage problem: $\operatorname{Cov}_{-} S \cup\left\{S_{i}\right\}$ covers any new target,

(2) for $k$-coverage problem: $\operatorname{Cov} S \cup\left\{S_{i}\right\}$ contributes to $k$-coverage requirement,

(3) for $Q$-coverage problem: $\operatorname{Cov}_{-} S \cup\left\{S_{i}\right\}$ contributes to $Q$-coverage requirement.

The detailed algorithm is discussed in Algorithm 1.

4.2. Cover Optimization. The cover may have nodes which need not be turned on for coverage condition to be satisfied. These nodes will be eliminated at this phase. The last node in Cov_S will be the one which completes the $1 / k / Q$ coverage requirement. Hence it will not be eliminated. Elimination check starts from the last but one node in Cov_S. It continues for all the other nodes in $\operatorname{Cov}_{-} S$ in the least priority first order. This curbs the possibility of higher priority nodes being eliminated at this stage. A node $S_{i} \in \operatorname{Cov}_{-} S, 1 \leq i \leq$ length $\left(\operatorname{Cov}_{-} S\right)$, represented as $S_{i} \cdot \operatorname{Cov}_{-} S$, will not be added to the optimized cover set Opt.Cov_S if $\operatorname{Cov}_{-} S-\left\{S_{i} \cdot \operatorname{Cov}_{-} S\right\}$ meets $1 / k / Q$ coverage requirement. The cover optimization algorithm is given in Algorithm 2.

4.3. M-Connected Cover Formation. The optimized cover is checked for $M$-connectivity. The connectivity matrix helps in finding out whether the nodes are $M$-connected or not. If the nodes are not $M$-connected, the nodes which got eliminated 


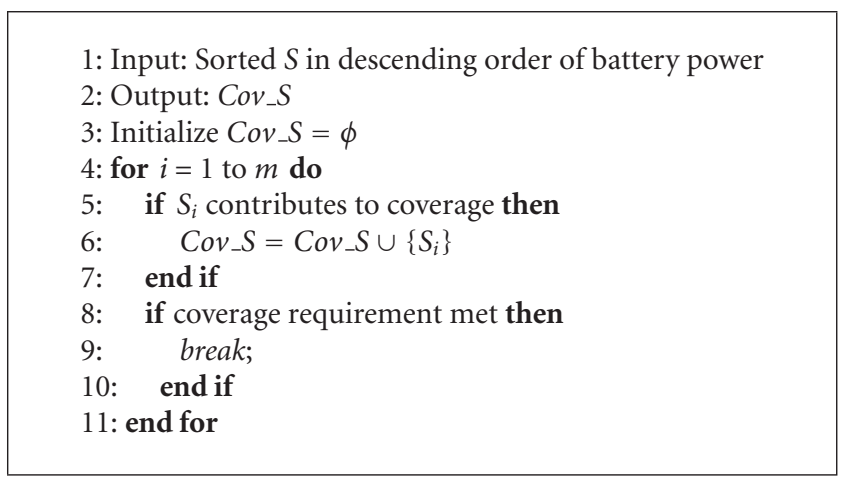

Algorithm 1: Cover formation.

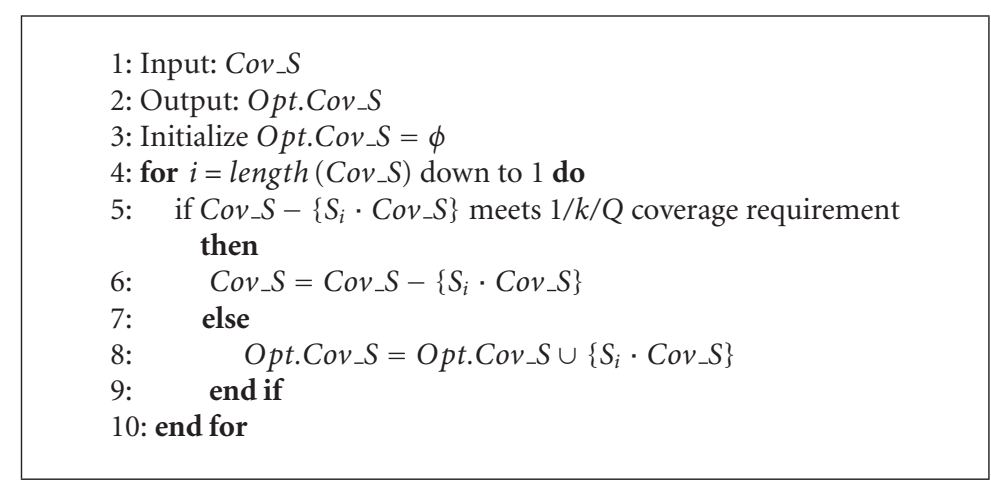

Algorithm 2: Cover optimization.

at the cover optimization phase and the nodes which did not form a part of Cov_S with battery power more than the minimum usable energy will also be considered. Since any node can play a vital role in making an $M$-connected cover, we add these remaining nodes one by one to the optimized cover and check for all possible $M$-connected subsets at each new addition. If any $M$-connected subset meets coverage requirement, this subset will be the $M$-connected cover. Algorithm 3 shows the formation of $M$-connected cover.

4.4. M-Connected Cover Optimization. A node $S_{i} \in M_{-}$Connected_Cover, $1 \leq i \leq$ length (M_Connected_Cover), is represented as $S_{i} \cdot M_{-}$Connected_Cover will not be added to the optimized cover set Opt.M_Connected_Cover if M_Connected_Cover $-\left\{S_{i} \cdot M_{-}\right.$Connected_Cover $\}$is $M$-connected and meets $1 / k / Q$ coverage requirement. $M$-connected cover optimization algorithm is given in Algorithm 4 .

\section{Results and Discussion}

We have considered a $200 \mathrm{~m} \times 200 \mathrm{~m}$ region for experiments. The number of sensors is varied between 150-250 to monitor 25 targets. The sensing range is fixed as $40 \mathrm{~m}$ and the communication range is $80 \mathrm{~m}$. Experiments are carried out for simple coverage, $k$ values $2,3,4$ and $Q$ values ranging from $1-3,1-4$, and $1-5 . M$ is assumed to take an integer value in the range of 1 to 3 .
5.1. Impact of Varying $k / Q$. When $k / Q$ requirement increases, the number of nodes that need to be turned on increases and since the number of nodes is large in each cover, there is a large possibility of the nodes being connected. This will leave the network lifetime unaffected when connectivity is also considered.

5.2. Impact of Varying $M$. For simple coverage problem, when $M$ increases, a slight decrease in network lifetime is observed (Figure 3). This is because only very few nodes need to be turned on for satisfying the coverage requirement. For making them $M$-connected, some other nodes will have to be turned on, bringing down the overall network lifetime. In case of higher $k / Q$ coverage requirement, since more numbers of nodes need to be turned on, there are chances that these nodes will be $M$-connected as well. Figures 4 and 5 show the network lifetime for $M$-connected $k$-coverage and $M$-connected $Q$-coverage, respectively.

5.3. Impact of Varying Number of Nodes. When average network lifetime is considered, for higher number of sensor nodes, network lifetime may or may not increase. The location of targets, location of sensors, and the $k / Q$ values contribute to determining the network lifetime. When given a region with more sensor nodes, it need not be necessary that the network lifetime will be high. If the region has more idle sensor nodes, there are chances that the network lifetime 


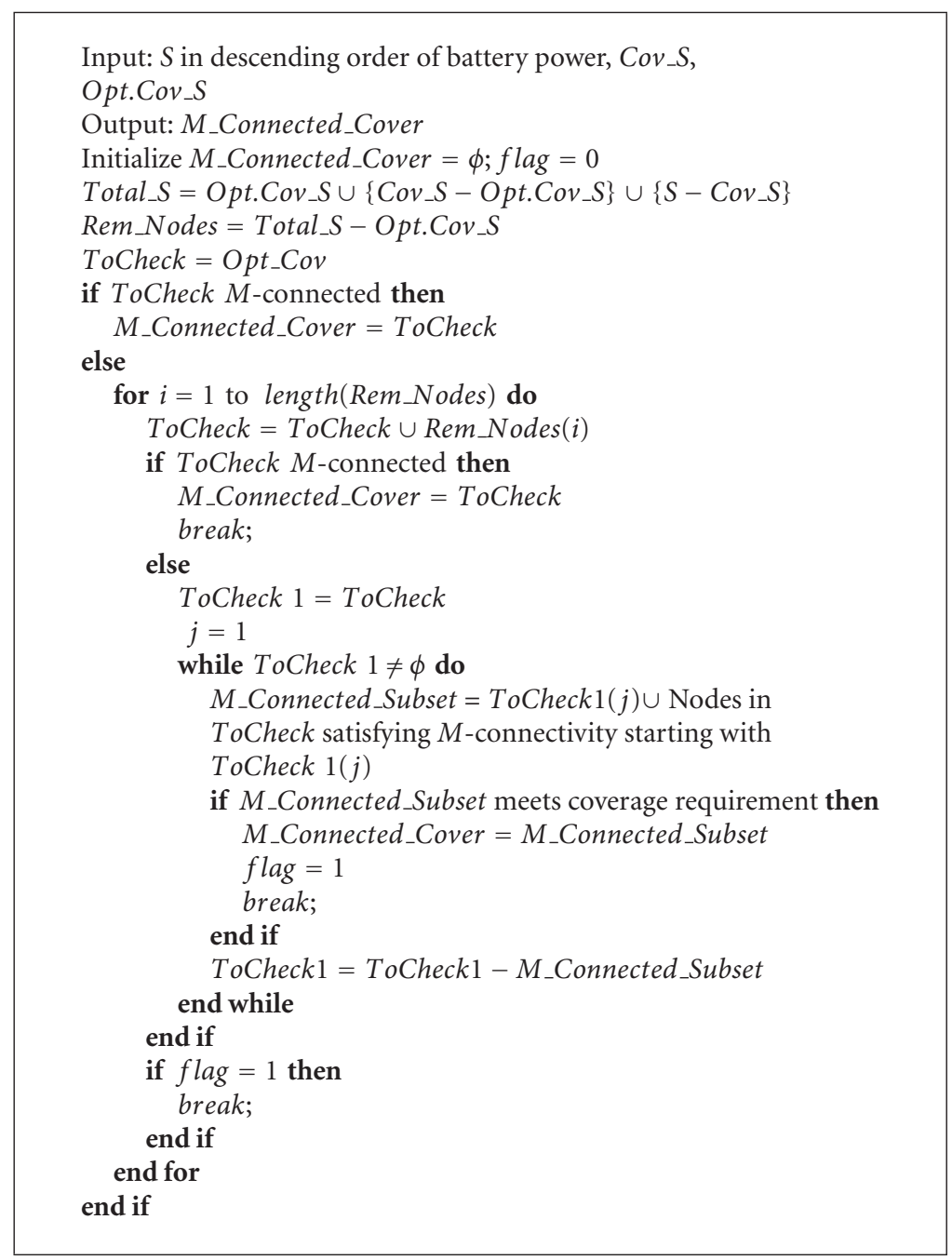

Algorithm 3: M-connected cover formation.

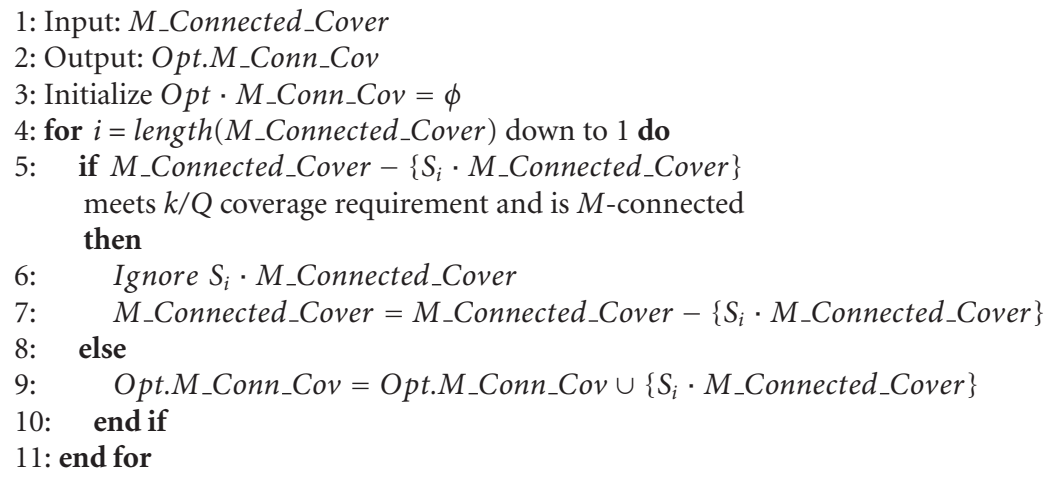

Algorithm 4: $M$-connected cover optimization.

may drop compared to a region with less number of sensor nodes where most of them are nonidle.

5.4. Comparison with CWGC. CWGC (Communication Weighted Greedy Cover) [21] uses a greedy method to sel- ect the set of sourcenodes (called source set) that cover the targets and it couples the communication cost and the selection of source sets. The problem modeled in CWGC requires that all the targets are covered by a subset of sensors and all the targets are connected to the sink node through a subset of 


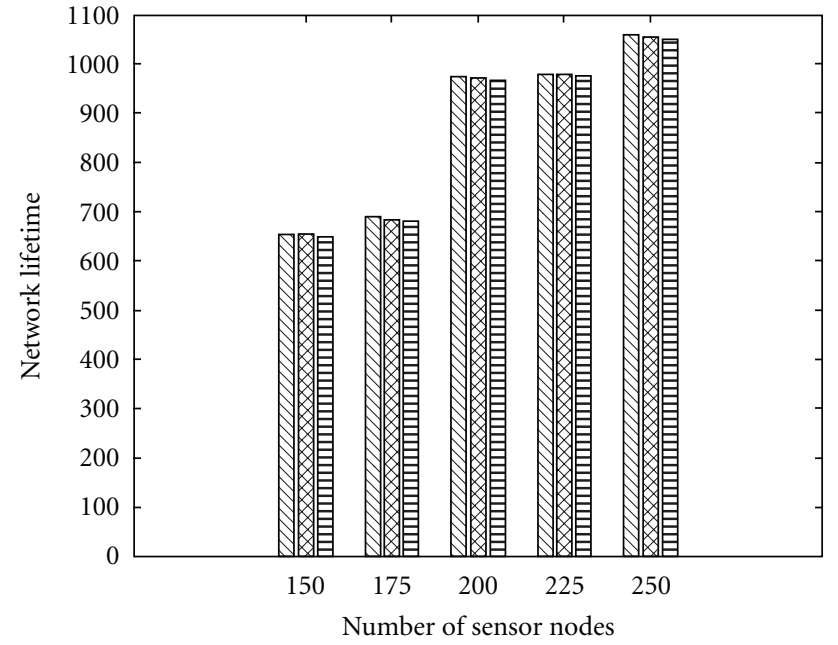

피 $M=1$
$M=2$
$M=3$

FIgURE 3: Network lifetime for $M$-connected simple coverage problem.

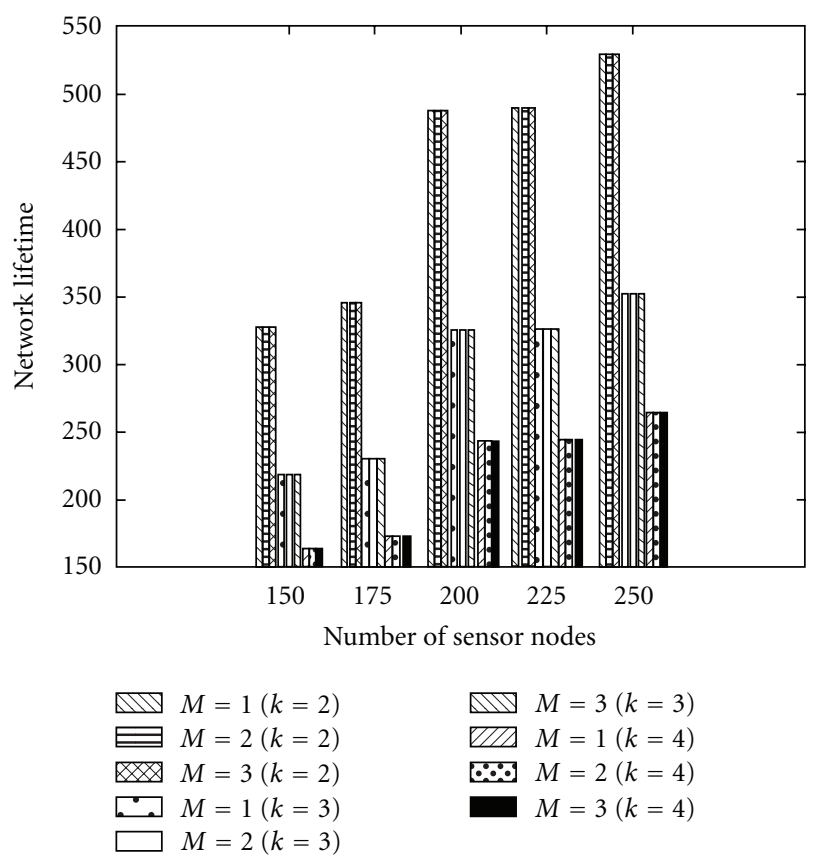

FIgURE 4: Network lifetime for $M$-connected $k$-coverage problem.

sensors by multihop paths. If any of the above requirements cannot be satisfied, the deployed WSN reaches its lifetime. The objective is to maximize the network lifetime of such a WSN. A dead sensor is a sensor which has no residual energy and an isolated sensor is one which has residual energy but cannot find a route from itself to the sink without traversing a dead sensor. The approach is to divide all the deployed sensors into a number of sensor sets each of which can
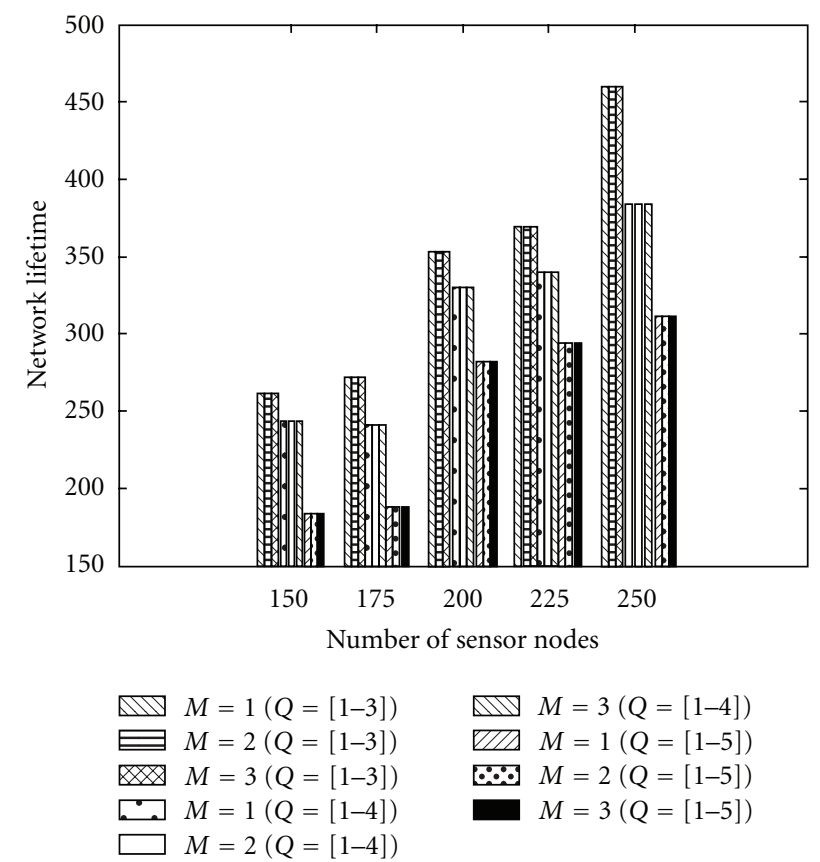

FIGURE 5: Network Lifetime for $M$-Connected $Q$-coverage problem.

cover all the targets and can send all the sensed data to the sink. These sensor sets need not be disjoint and are activated successively one by one. Before each iteration of building a new cover tree, the dead sensors and isolated sensors in the network will be removed. Only sensors in an active set are used to sense targets and to relay data to the sink, and all the other sensors go into an energy saving sleep state.

Though CWGC is for multihop communication where a sensor cannot reach the sink node directly, and many of the assumptions do not match our model, we have done a comparison based on the operational time of a sensor cover in CWGC. In CWGC, each cover operates for a fixed time duration, unless some sensors in the cover will die before the end of the time duration due to the lack of energy. This might not give the optimal network lifetime for some cases. We have shown this with an example in Figure 6 for $k$-coverage problem with $k=2$. The region has 3 sensor nodes $S_{1}, S_{2}$, $S_{3}$ with battery power 100 units and energy consumption rate 1 unit, and two targets $T_{1}$ and $T_{2}$. Each sensor node is able to monitor both the targets and all the three sensor nodes are connected. Let the initial cover formed be $\left\{S_{1}, S_{2}\right\}$. With CWGC, the same cover will be active till at least one node dies. So, the cover will be active for 100 units of time, yielding a network lifetime of 100 units. With our proposed method, if covers are computed for each time instant, based on priority of sensor node (battery power), the following will be the sensor covers $\left\{\left\{S_{1}, S_{2}\right\},\left\{S_{3}, S_{1}\right\},\left\{S_{2}, S_{3}\right\}, \ldots\right\}$. This will give a network lifetime of 150 units. Figure 7 shows a comparison of our proposed approach with CWGC for simple coverage problem. Figures 8 and 9 show a comparison of our proposed approach with CWGC for $k$-coverage and $Q$-coverage problems, respectively, together with $M$ connectivity. 


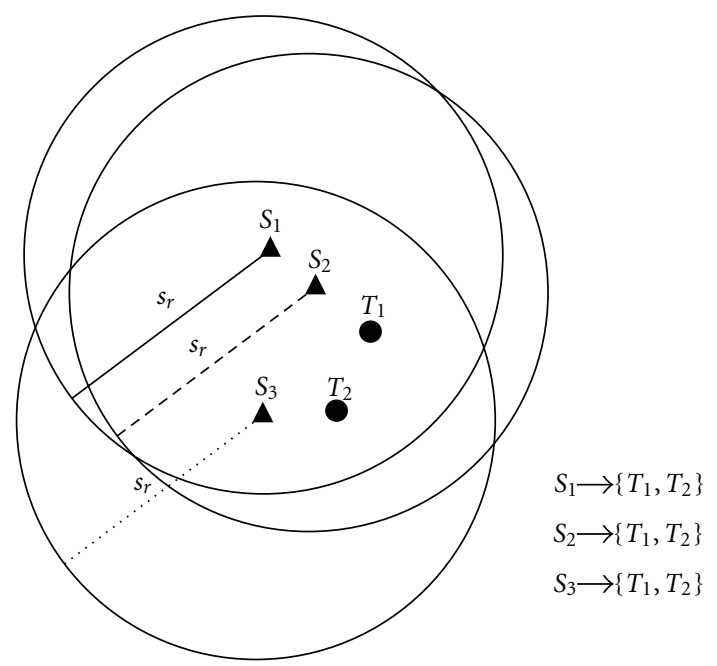

FIgURE 6: An example.

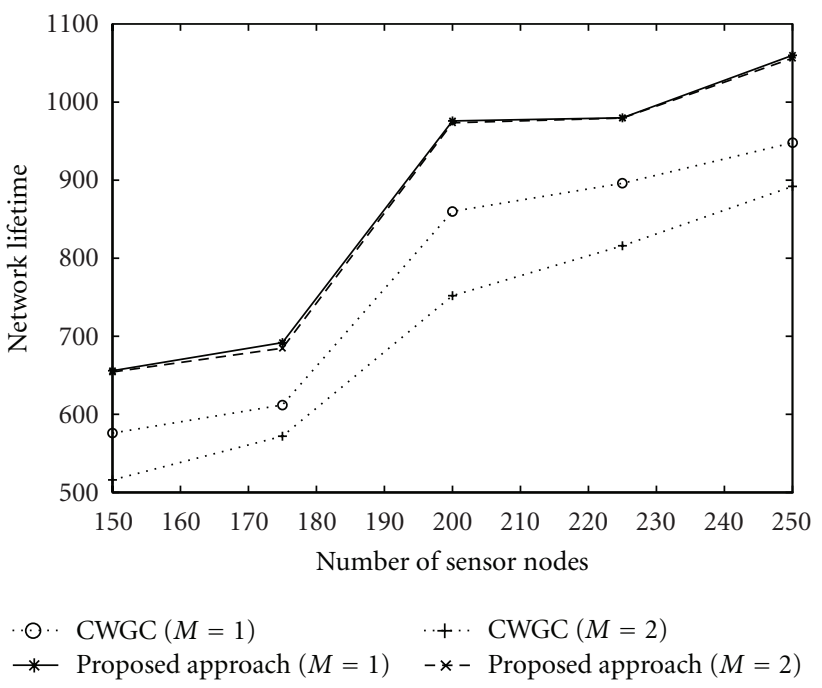

FIGURE 7: Comparison of CWGC and proposed approach for $M$ connected simple coverage problem.

\section{Conclusions and Future Work}

Sensitive applications of wireless sensor networks require high level of connectivity as well as coverage. We propose a method to schedule the sensor nodes such that only minimum number of sensor nodes will be active, satisfying connectivity and coverage requirement. This leads to higher network lifetime. The need for such a method arises when all the targets need not be monitored with the same proximity and when $M$-connectedness should exist within the nodes that are turned on, to guarantee the correctness of the information collected and also to ensure the reachability of the information at other nodes including base station. We observe that the introduction of connectivity does not affect the network lifetime to a greater extent. The proposed method performed better than CWGC. In future, we plan to

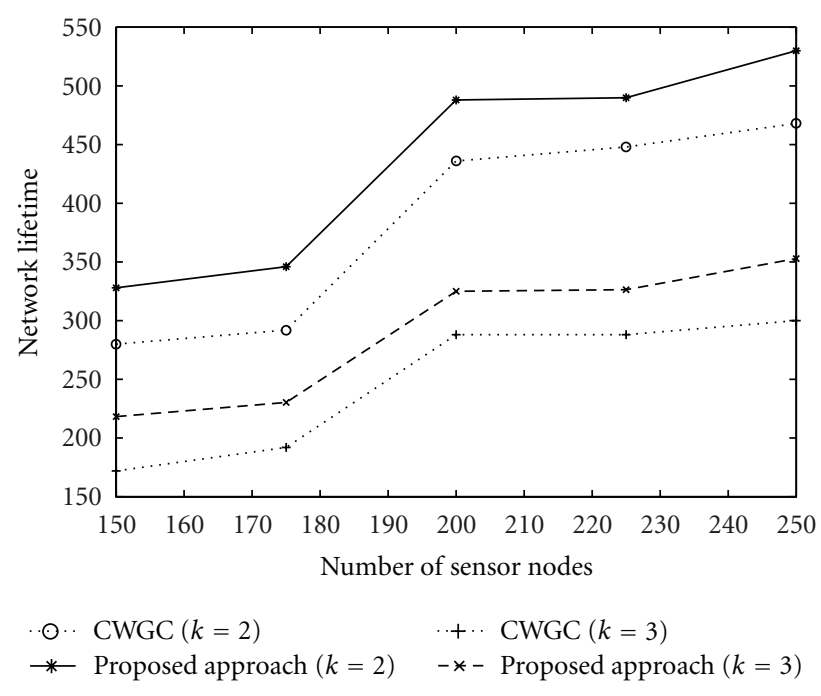

Figure 8: Comparison of CWGC and proposed approach for $M$ connected $k$-coverage problem where $M=2$.

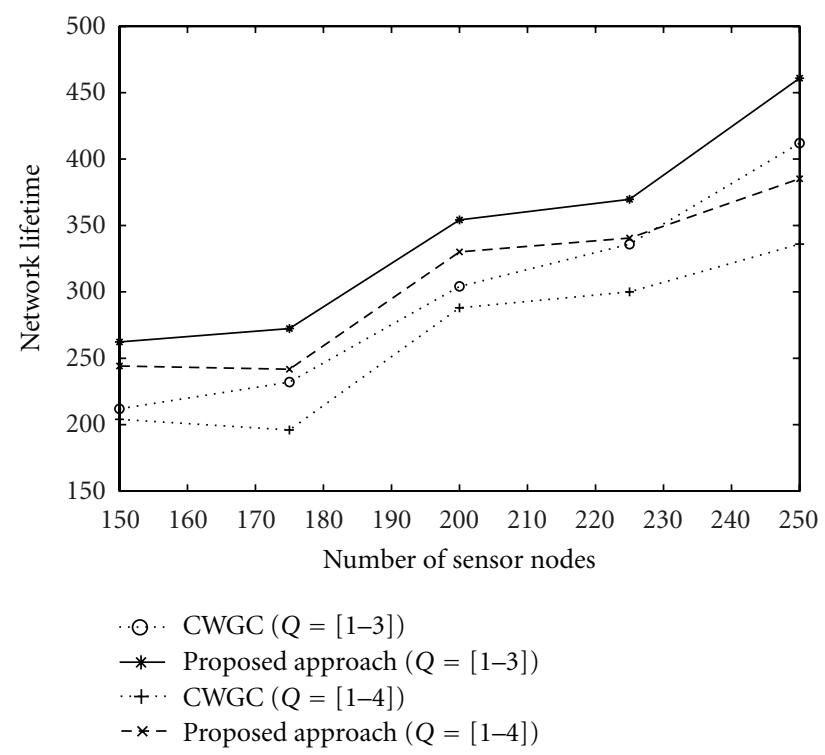

FIGURE 9: Comparison of CWGC and proposed approach for $M$ connected $Q$-coverage problem where $M=2$.

extend this work for mobile wireless sensor network environment.

\section{References}

[1] J. Yick, B. Mukherjee, and D. Ghosal, "Wireless sensor network survey," Computer Networks, vol. 52, no. 12, pp. 2292-2330, 2008.

[2] E. Jain and Q. Liang, "Sensor placement and lifetime of wireless sensor networks: theory and performance analysis," in Proceedings of the IEEE Global Telecommunications Conference (GLOBECOM '05), pp. 173-177, St. Louis, Mo, USA, December 2005 . 
[3] Z. Liu, "Maximizing network lifetime for target coverage problem in heterogeneous wireless sensor networks," in Proceedings of the 3rd International Conference on Mobile Ad-Hoc and Sensor Networks (MSN '07), pp. 457-468, Beijing, China, December 2007.

[4] W. R. Heinzelman, A. Chandrakasan, and H. Balakrishnan, "Energy-efficient communication protocol for wireless microsensor networks," in Proceedings of the 33rd Annual Hawaii International Conference on System Siences (HICSS '00), pp. 3005-3014, Maui, Hawaii, USA, January 2000.

[5] D. G. Costa and L. A. Guedes, "The coverage problem in video-based wireless sensor networks: a survey," Sensors, vol. 10, no. 9, pp. 8215-8247, 2010.

[6] J. N. Al-Karaki and A. E. Kamal, "Routing techniques in wireless sensor networks: a survey," IEEE Wireless Communications, vol. 11, no. 6, pp. 6-28, 2004.

[7] J. Chen and X. Koutsoukos, "Survey on coverage problems in wireless ad hoc sensor networks," in Proceedings of the IEEE SouthEast Conference, pp. 22-25, Richmond, Va, USA, March 2007.

[8] Y. Li and S. Gao, "Designing $k$-coverage schedules in wireless sensor networks," Journal of Combinatorial Optimization, vol. 15, no. 2, pp. 127-146, 2008.

[9] C. F. Huang and Y. C. Tseng, "The coverage problem in a wireless sensor network," in Proceedings of the 2nd ACM International Workshop on Wireless Sensor Networks and Applications (WSNA '03), pp. 115-121, San Diego, Calif, USA, September 2003.

[10] Y. Gu, H. Liu, and B. Zhao, "Target coverage with QoS requirements in wireless sensor networks," in Proceedings of the International Conference on Intelligent Pervasive Computing (IPC '07), pp. 35-38, Washington, DC, USA, October 2007.

[11] S. Slijepcevic and M. Potkonjak, "Power efficient organization of wireless sensor networks," in Proceedings of the IEEE International Conference on Communications (ICC '01), pp. 472476, Helsinki, Finland, June 2001.

[12] M. Cardei and D. Z. Du, "Improving wireless sensor network lifetime through power aware organization," Wireless Networks, vol. 11, no. 3, pp. 333-340, 2005.

[13] M. Cardei, M. T. Thai, Y. Li, and W. Wu, "Energy-efficient target coverage in wireless sensor networks," in Proceedings of the IEEE International Conference on Computer Communications (INFOCOM '05), pp. 1976-1984, Miami, Fla, USA, March 2005.

[14] M. Hefeeda and M. Bagheri, "Randomized $k$-coverage algorithms for dense sensor networks," in Proceedings of the 26th IEEE International Conference on Computer Communications (INFOCOM '07), pp. 2376-2380, Anchorage, Alaska, USA, May 2007.

[15] M. Chaudhary and A. K. Pujari, "Q-coverage problem in wireless sensor networks," in Proceedings of the 10th International Conference on Distributed Computing and Networking (ICDCN '09), pp. 325-330, Hyderabad, India, January 2009.

[16] Y. Gu, Y. Ji, J. Li, and B. Zhao, "QoS-aware target coverage in wireless sensor networks," Wireless Communications and Mobile Computing, vol. 9, no. 12, pp. 1645-1659, 2009.

[17] Y. Gu, B.-H. Zhao, Y.-S. Ji, and J. Li, “Theoretical treatment of target coverage in wireless sensor networks," Journal of Computer Science and Technology, vol. 26, no. 1, pp. 117-129, 2011.

[18] Z. Zhou, S. Das, and H. Gupta, "Connected $k$-coverage problem in sensor networks," in Proceedings of the 13th International Conference on Computer Communications and Networks (ICCCN '04), pp. 373-378, Chicago, Ill, USA, October 2004.
[19] M. Lu, J. Wu, M. Cardei, and M. Li, "Energy-efficient connected coverage of discrete targets in wireless sensor networks," in Proceedings of the 3rd International Conference on Computer Network and Mobile Computing (ICCNMC '05), pp. 43-52, Zhangjiajie, China, August 2005.

[20] H. Gupta, S. R. Das, and Q. Gu, "Connected sensor cover: selforganization of sensor networks for efficient query execution," in Proceedings of the 4th ACM International Symposium on Mobile Ad Hoc Networking and Computing (MobiHoc '03), pp. 189-200, Annapolis, Md, USA, June 2003.

[21] Q. Zhao and M. Gurusamy, "Lifetime maximization for connected target coverage in wireless sensor networks," IEEE/ACM Transactions on Networking, vol. 16, no. 6, pp. 1378-1391, 2008.

[22] X. Bai, Z. Yun, D. Xuan, T. H. Lai, and W. Jia, "Deploying four-connectivity and full-coverage wireless sensor networks," in Proceedings of the 27th IEEE Communications Society Conference on Computer Communications (INFOCOM '08), pp. 296-300, Phoenix, Ariz, USA, April 2008.

[23] Y. C. Wang, C. C. Hu, and Y. C. Tseng, "Efficient deployment algorithms for ensuring coverage and connectivity of wireless sensor networks," in Proceedings of the 1st International Conference on Wireless Internet (WICON '05), pp. 114-121, Budapest, Hungary, July 2005.

[24] H. M. Ammari and S. K. Das, "Clustering-based minimum energy wireless $m$-connected $k$-covered sensor networks," in Proceedings of the 5th European Conference on Wireless Sensor Networks (EWSN '08), pp. 1-16, Bologna, Italy, January 2008.

[25] X. Han, X. Cao, E. L. Lloyd, and C. C. Shen, "Deploying directional sensor networks with guaranteed connectivity and coverage," in Proceedings of the 5th Annual IEEE Communications Society Conference on Sensor, Mesh and Ad Hoc Communications and Networks (SECON '08), pp. 153-160, San Francisco, Calif, USA, June 2008.

[26] Q. Gao and H. Zou, "Improving probabilistic coverage and connectivity in wireless sensor networks: cooperation and mobility," in Proceedings of the International Conference on Wireless Communications and Signal Processing (WCSP '10), pp. 1-6, Suzhou, China, 2010.

[27] A. Krause, C. Guestrin, A. Gupta, and J. Kleinberg, "Near-optimal sensor placements: maximizing information while minimizing communication cost," in Proceedings of the 5th International Conference on Information Processing in Sensor Networks (IPSN '06), pp. 2-10, Nashville, Tenn, USA, April 2006.

[28] K. Kar and S. Banerjee, "Node placement for connected coverage in sensor networks," in Proceedings of the 1st International Symposium on Modeling and Optimization in Mobile, Ad-Hoc and Wireless Networks (WiOpt '03), Sophia-Antipolis, France, March 2003. 

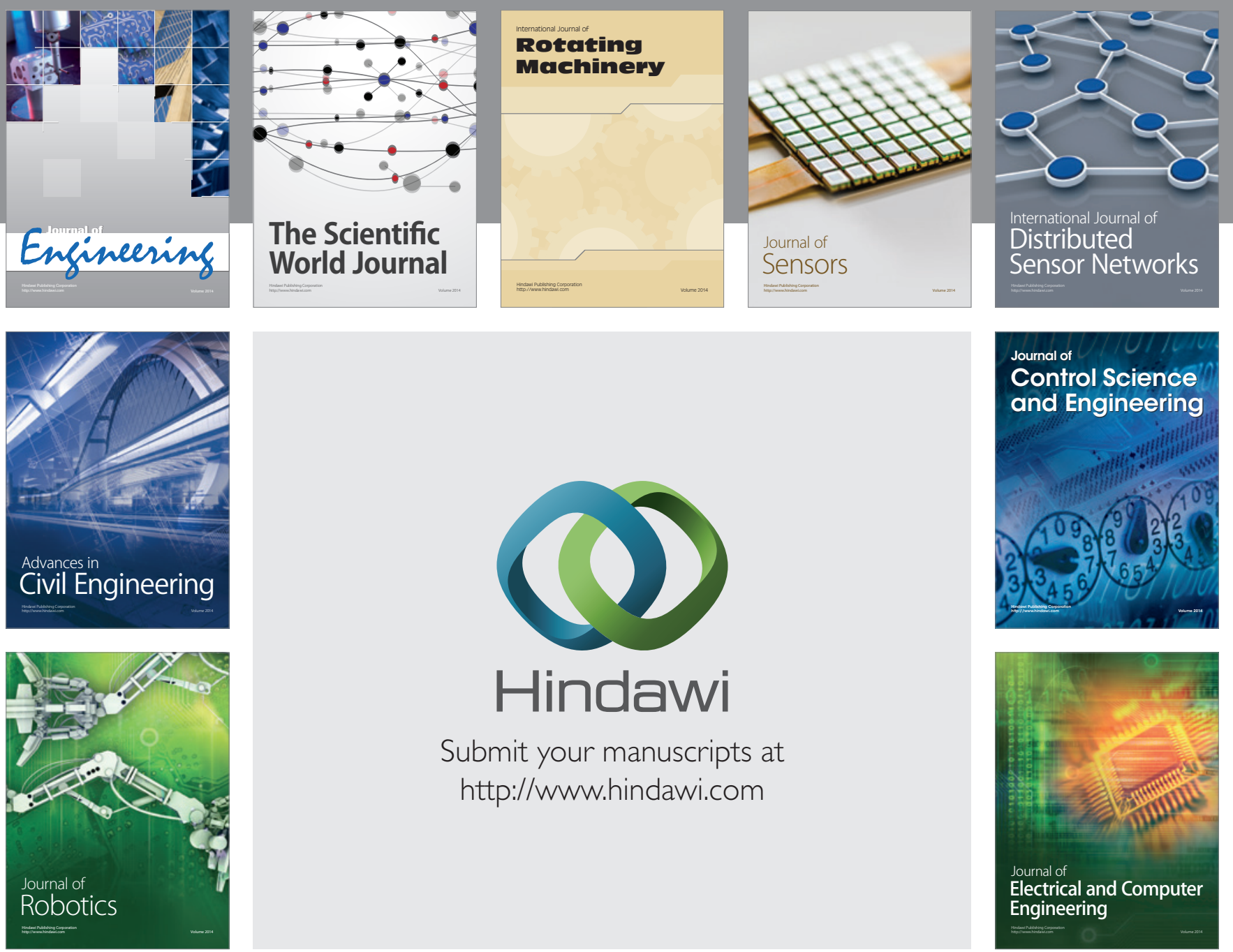

Submit your manuscripts at

http://www.hindawi.com
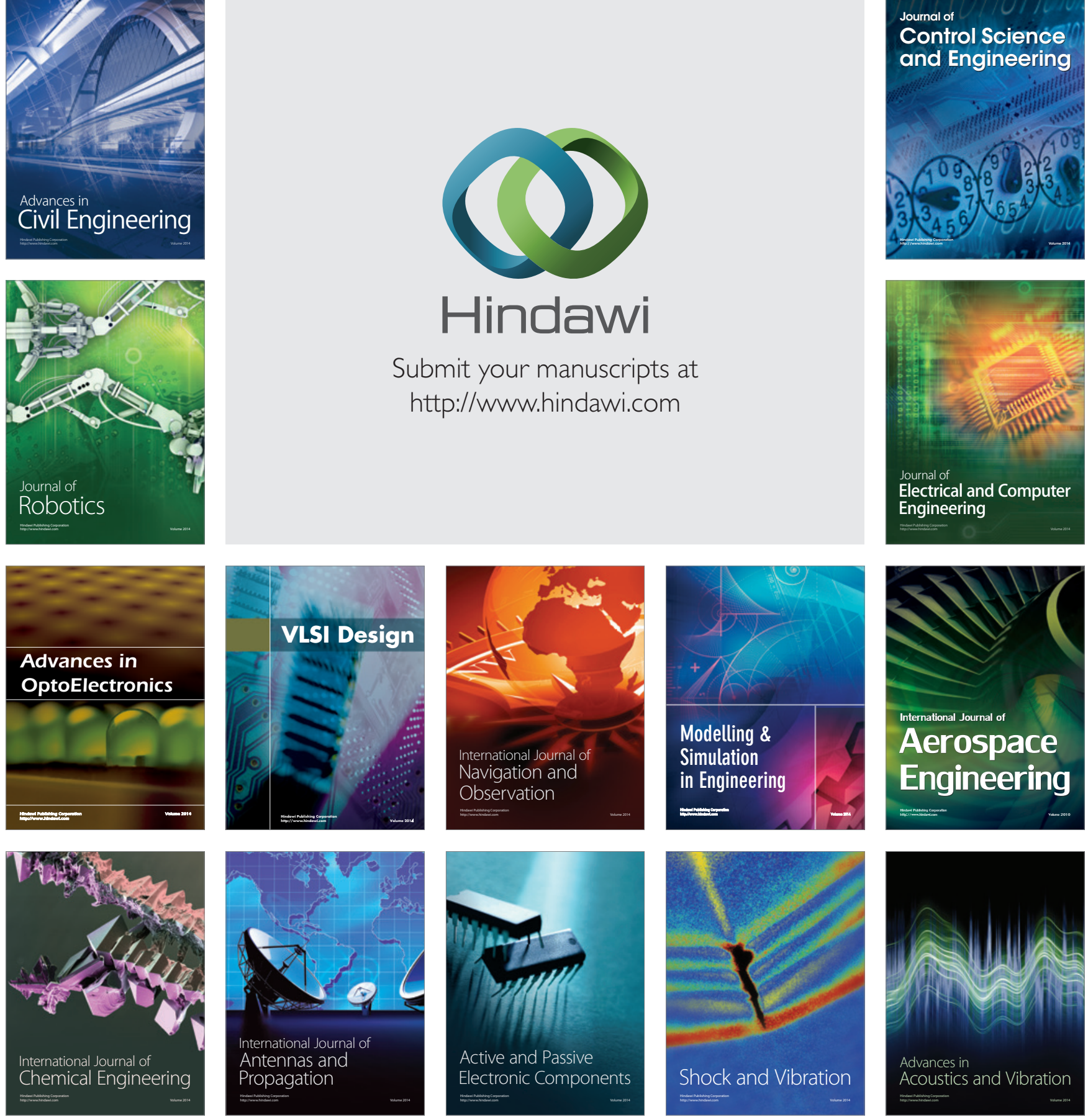